\title{
MODELAGEM MATEMÁTICA APLICADA NO PLANEJAMENTO DE PESQUISA DE TRÁFEGO RODOVIÁRIO
}

\section{MATHEMATICAL MODELING APPLIED TO THE ROAD TRAFFIC SURVEY PLANNING}

\author{
Marcus Vinicius Oliveira Camara*E-mail: marcus@pet.coppe.ufri.br \\ Glaydston Mattos Ribeiro*E-mail: glaydston@pet.coppe.ufri.br \\ Saul Germano Rabelo Quadros* E-mail: saul quadros@yahoo.com.br \\ Cel Alexandre da Hora** E-mail: alexandre.hora@uol.com.br \\ Cel Jorge Fernando do Nascimento*** E-mail: jorge@coter.eb.mil.br \\ Carlos Alberto Abramides ${ }^{* * *}$ E-mail: carlos.abramides@dnit.gov.br \\ *Universidade Federal do Rio de Janeiro (UFRJ), Rio de Janeiro, RJ \\ **Exército Brasileiro - Colégio Militar de Brasília, Brasília, DF \\ ${ }^{* * \star}$ Exército Brasileiro - Comando de Operações Terrestres, Brasília, DF \\ ****Departamento Nacional de Infraestrutura de Transportes, Brasília, DF
}

\begin{abstract}
Resumo: O planejamento estratégico do setor de transportes indica as principais diretrizes para investimentos em infraestrutura de maneira a combater, por exemplo, o aumento dos custos associados aos deslocamentos. Com isso, torna-se relevante, minimamente, o conhecimento relativo aos volumes médios diários de tráfego nas rodovias, cargas transportadas, origem e destino de viagens. Estes dados são obtidos por meio de pesquisas de contagem volumétrica e classificatória e de origem e destino. Atualmente, o Departamento Nacional de Infraestrutura de Transportes (DNIT) planeja uma nova pesquisa nacional de tráfego em 300 pontos de pesquisa. Historicamente, o Exército Brasileiro (EB) tem apoiado esta atividade por meio de suas Organizações Militares (OM). Sendo assim, este artigo apresenta um modelo matemático para localização-alocação de OM a postos de pesquisa rodoviária, considerando as características operacionais (capacidade e disponibilidade) e locacionais (abrangência de atendimento) de cada OM. Além disso, foram avaliados cenários simulados que permitem avaliar a aplicação do modelo em diferentes combinações de parâmetros.
\end{abstract}

Palavras-chave: Localização de Facilidades. Modelagem. Transportes. Pesquisa de Tráfego.

Abstract: The strategic planning of the transportation sector indicates the main guidelines for infrastructure investments to avoid, for example, the increase in costs associated with transports. Therefore, it is relevant to know the annual average daily traffic on roads, cargo transported, origin and destination of the vehicles. These data are obtained through traffic surveys, which include flows and origin and destination. Currently, the National Department of Transport Infrastructure plans a new national traffic survey in 300 points. Historically, the Brazilian Army has supported this activity by Military Organizations (OM). Thus, this paper presents a mathematical model for location-allocation of $\mathrm{OM}$ to traffic survey stations, taking into account constraints, which consider operational characteristics (capacity and availability) and spatial locations (covering) of each OM. Moreover, scenarios are proposed to evaluate the model in different combinations of parameters.

Keywords: Facility Location. Modeling. Transportation. Traffic Survey. 


\section{INTRODUÇÃO}

Fundamental para o desenvolvimento econômico e aumento da competitividade de uma nação, o planejamento estratégico do setor de transportes indica as principais diretrizes para investimentos em infraestrutura, tanto na ampliação quanto implantação, de maneira a reduzir os custos, tempos de viagens e outras ineficiências relacionadas à ausência de planejamento e investimentos no setor.

Com isso, torna-se relevante o conhecimento relativo aos volumes médios diários de tráfego nas rodovias, informações de predominância de cargas transportadas e de veículos, suas respectivas sazonalidades relacionadas, características de motivação, origem e destino de viagens, entre outras, que permitem o desenvolvimento de atividades que envolvem planejamento e calibração de fluxo de transportes.

Por outro lado, a obtenção dessas informações e conhecimento dessas características peculiares do setor necessita de mecanismos constantes de coleta de dados. No entanto, o desenvolvimento desses mecanismos, de forma permanente, pode ser altamente custoso, dos pontos de vista financeiro e operacional, o que pode resultar em prejuízos técnicos no planejamento estratégico de transportes.

A utilização de dados provenientes de pedágios rodoviários, por exemplo, que embora sejam pontuais, podem ser utilizados para essa finalidade, porém, quando se trata de um macroplanejamento (nível nacional), o Brasil, que possui considerável diversidade socioeconômica e cultural, e principalmente, grande extensão territorial associada, necessita de mecanismos específicos e mais eficientes.

Assim, a aplicação de pesquisas de tráfego rodoviário faz-se necessário de forma a subsidiar, além de estudos de viabilidade econômica e projetos de engenharia, o planejamento estratégico de transportes que englobe as características das distintas regiões do país, e permita a análise da rede de transportes como um todo.

Nesse sentido, no ano de 1975, iniciou-se a utilização de pesquisas de contagem de tráfego no Brasil com o Programa de Contagem Sistemática de Trânsito, implantada nos Estados do RJ, SP e MG. Em 1976, o programa anterior 
deu origem ao Plano de Contagem Sistemática de Trânsito, e devido ao sucesso da implantação desses, já em 1977, iniciou-se o Plano Nacional de Contagem de Trânsito que contou com 120 postos de contagem permanente (DNIT, 2015).

Durante os anos seguintes, as pesquisas de contagem de tráfego, em busca de maior abrangência, utilizaram 235 equipamentos de contagem em 1989, 266 em 1997, e no ano de 1998 chegou ao ápice com 285 postos distribuídos no território nacional. Durante a década seguinte, o programa foi interrompido por questões de restrições orçamentárias governamentais.

Por outro lado, a necessidade de informações para o planejamento estratégico de transportes ultrapassa dados provenientes de contagem volumétrica de veículos. Além desses, é muito relevante à obtenção de informações relacionadas às origens e destinos das viagens, características de cargas transportadas, motivação das viagens e outros.

Nesse contexto, o Departamento Nacional de Infraestrutura de Transportes (DNIT, 2015) promoveu a realização de novas pesquisas de tráfego envolvendo o Plano Direto Nacional Estratégico de Pesagem, realizado em 2005, e o Plano de Investimento em Transportes, realizado em 2006, para os eixos de escoamento da soja, conhecido como Operação Safra.

Em 2008, de maneira similar, foi realizada uma pesquisa no Polo Rodoviário de Pelotas, dessa vez com objetivo específico de auxiliar no desenvolvimento de estudos de impactos relacionados ao processo de concessão rodoviária do trecho. Por fim, em 2011 realizou-se o que pode ser considerada como a maior pesquisa de tráfego em território nacional, conhecida como Pesquisa Nacional de Tráfego - PNT (MT, 2011).

Na PNT de 2011, foram realizadas de maneira simultânea, Pesquisa de Contagem Volumétrica e Classificatória (CVC) e de Origem e Destino (O/D), em 120 postos de pesquisa distribuídos em todo território nacional. No entanto, pesquisas com tal magnitude e complexidades logística e operacional relacionadas, necessitam, entre outros fatores, de mão-de-obra adequada.

Nesse sentido, desde a pesquisa de 2005, o DNIT contou com a atuação do Exército Brasileiro (EB), por intermédio de suas Organizações Militares (OM), no suporte e na realização das pesquisas de tráfego. É importante destacar que a capacidade operacional do EB, sua ampla distribuição no território nacional, além da 
vasta experiência em operações de campo se mostraram importantes na eficiência e êxito das pesquisas realizadas. Na PNT de 2011, foram utilizados mais de 5.000 homens do EB.

Atualmente, o DNIT planeja realizar outra PNT, em parceria com a Universidade Federal do Rio de Janeiro (UFRJ) e o EB, que contempla 300 postos de pesquisa CVC e O/D e que, espera-se empregar no máximo 120 OM. Cada posto de pesquisa deverá ser ocupado por uma tropa valor pelotão do Exército Brasileiro, com um efetivo aproximado de 42 homens. O Pelotão é uma tropa com efetivo variável da ordem de 36 a 50 homens, comandado por 01 (um) oficial subalterno (Tenente). Os pelotões formam companhias (normalmente cada companhia possui 03 pelotões), e estas formam os Batalhões.

Diante da magnitude desta nova PNT e dos parâmetros operacionais, buscouse trabalhar com distâncias compatíveis a fim de se diminuir o tempo de deslocamento dos pelotões até os postos de pesquisa. De maneira geral, os militares deslocam-se até os postos de pesquisa e permanecem por lá até o final do período que é estimado em uma semana. As pesquisas são realizadas durante 24 horas por dia.

Diante do que foi apresentado, apresenta-se o Problema de LocalizaçãoAlocação de Organizações Militares a Postos de Pesquisa (PL-AOMPP) que deve determinar quais OM serão utilizadas, e ainda, a quais postos de pesquisa estas deverão ser alocadas de maneira a minimizar o tempo total de deslocamento das tropas de cada OM aos respectivos postos alocados.

Dessa forma, o presente estudo tem como objetivo principal apresentar um modelo matemático para localização-alocação de $\mathrm{OM}$ a postos de pesquisa rodoviária de tráfego no território nacional, considerando as características operacionais (capacidade e disponibilidade) e locacionais (abrangência de atendimento) de cada OM.

Quanto aos objetivos, esta pesquisa pode ser considerada exploratória por utilizar pesquisas bibliográficas com finalidade de ampliar a compreensão do tema abordado. Quanto aos procedimentos técnicos, utilizou-se pesquisa documental com objetivo de analisar e melhor compreender materiais que não receberam tratamento analítico a priori. Além disso, a pesquisa pode ser considerada um estudo de caso 
por apresentar uma investigação de fenômenos contemporâneos em seu contexto real.

O restante do artigo está dividido da seguinte forma: na Seção 2 é apresentada uma revisão bibliográfica acerca de modelos de localização-alocação; o modelo matemático desenvolvido para o PL-AOMPP apresenta-se na Seção 3; os resultados dos experimentos computacionais mostram-se na Seção 4; e por fim, na Seção 5 estão as considerações finais do estudo.

\section{REVISÃO BIBLIOGRÁFICA}

Dentro da grande área Pesquisa Operacional, o Problema de Localização de Facilidades (PLF) mostra-se como importante linha de pesquisa, onde a denominada "facilidade" relaciona-se com elementos de localização fixa distantes desta. Dessa forma, a relação de distância entre as facilidades que devem ser localizadas e os demais elementos que devem ser atendidos, além de outros fatores, torna-se determinante na escolha da função objetivo a ser otimizada.

O termo "facilidade" representa instalações físicas de suporte, como fábricas, depósitos, escolas, antenas e outros. Dessa forma, o PLF enunciado pode ser representado por meio de redes que são compostas por pontos, também chamados de nós ou vértices, e linhas, também conhecidas como arcos ou arestas, e assim, podem descrever vias públicas e outros (ARAKAKI, 2003).

O PLF também é conhecido como Problema de Localização-Alocação de Facilidades (PL-AF), isso porque, em algumas situações reais, ao determinar a localização das facilidades, deve-se também, indicar sua área de atendimento, ou seja, quais elementos devem ser atendidos por cada facilidade.

No entanto, as aplicações e teoria relacionadas a problemas de localização são extensas e envolvem fatores distintos como: área de implementação; acessibilidade; disponibilidade e custo da matéria-prima; utilidades públicas; questões mercadológicas; aspectos ambientais e físico-geográficos; ocupação urbana; recursos humanos; incentivos fiscais; entre outros (BALLOU, 2001; CHUANG, 2001; GALVÃO et al., 2003; LOPEZ e HENDERSON, 1989; ROMERO, 2006; YANG e LEE, 1997) o que indica o surgimento de diferentes modelos matemáticos, com características específicas a cada problemática. 
Assim, diferentes classificações para problemas de localização são apresentadas na literatura: Modelos Planares e de Rede; Modelos Discretos; Modelos Estáticos e Dinâmicos; Modelos de Único e Multi Produtos; Modelos Determinísticos e Estocásticos; Modelos Mono e Multi Objetivos; e Modelos Capacitados e Não-Capacitados (BRANDEAU e CHIU, 1989; KRARUP e PRUZAN, 1990). O Quadro 1 apresenta um resumo das classificações indicadas.

Quadro 1 - Resumo de classificações para problemas de localização

\begin{tabular}{|c|l|}
\hline Modelos & \multicolumn{1}{c|}{ Descrição } \\
\hline $\begin{array}{c}\text { Planares e de } \\
\text { Rede }\end{array}$ & $\begin{array}{l}\text { De acordo com Klose e Drexl (2005), nos modelos planares, as instalações } \\
\text { (facilidades) e os demais pontos podem ser representados "espalhados" em } \\
\text { qualquer localização do plano. }\end{array}$ \\
\hline Discretos & $\begin{array}{l}\text { Nesses modelos, normalmente, considera-se que os pontos que representam } \\
\text { as instalações e os pontos de demanda de atendimento estão localizados em } \\
\text { pontos espećficos de uma rede, denominada grafo (HURTER e MARTINICH, } \\
1989 ; \text { DASKIN, 1995). }\end{array}$ \\
\hline $\begin{array}{c}\text { Estáticos e } \\
\text { Dinâmicos }\end{array}$ & $\begin{array}{l}\text { Quando os dados, ou parâmetros, que representam o problema não são } \\
\text { dependentes do tempo, ou seja, uma análise é feita para um período específico, } \\
\text { os modelos são classificados como Estáticos. Contudo, se os parâmetros } \\
\text { sofrem variações em função do tempo, os modelos são Dinâmicos. }\end{array}$ \\
\hline $\begin{array}{c}\text { Único e Multi } \\
\text { Produtos }\end{array}$ & $\begin{array}{l}\text { Segundo Nogueira (2007), a grande maioria dos modelos de localização tratam } \\
\text { de facilidades que, de maneira generalizada, atendem a um único produto. No } \\
\text { entanto, é possível que as instalações devam atender às demandas de } \\
\text { diferentes serviços (produtos). }\end{array}$ \\
\hline $\begin{array}{c}\text { Determinísticos } \\
\text { e Estocásticos }\end{array}$ & $\begin{array}{l}\text { Nos modelos Determinísticos, os parâmetros envolvidos, como demanda por } \\
\text { serviços e localização dos pontos de atendimento são conhecidos, por outro } \\
\text { lado, os parâmetros podem ser tratados de forma probabilística. }\end{array}$ \\
\hline $\begin{array}{c}\text { Mono e Multi } \\
\text { Objetivos }\end{array}$ & $\begin{array}{l}\text { Nos modelos Multiobjetivo, pode ser de interesse que mais de uma função } \\
\text { objetivo seja otimizada simultaneamente. Nesses modelos minimizar o custo } \\
\text { total (instalação da facilidade e transporte para os pontos de demanda), objetivo } \\
\text { comum nos modelos mono objetivo, pode estar associado a outros, como } \\
\text { maximizar a cobertura ou o nível de serviço, de maneira simultânea. }\end{array}$ \\
\hline $\begin{array}{c}\text { Capacitados e } \\
\text { Não- }\end{array}$ & $\begin{array}{l}\text { As facilidades (instalações) podem ser representadas com capacidades } \\
\text { ilimitadas (Não-Capacitados) ou então, podem ter uma capacidade de } \\
\text { atendimento associada (Capacitados). }\end{array}$ \\
\hline Capacitados
\end{tabular}

Além disso, os PLFs, e seus modelos matemáticos associados, são classificados na literatura como modelos de localização de Cobertura, $p$-Centro e $p$ Medianas (CRAINIC e LAPORTE, 1997; DUBKE, 2006; DREZNER e HAMACHER, 2002; MAPA 2007). Nos problemas de Máxima Cobertura, as facilidades estão associadas à sua cobertura de atendimento, ou seja, as instalações estão associadas a uma distância crítica, de forma que, a facilidade cobre o ponto de atendimento, se, e somente se, a distância entre o ponto de demanda for menor que a distância crítica. 
Para Dubke (2006) e Drezner e Hamacher (2002), os problemas de Cobertura podem ser representados por dois modelos matemáticos: o problema de localização para cobertura de conjuntos; e o problema de localização de máxima cobertura. No primeiro, deve-se determinar o número mínimo de instalações e suas respectivas localizações para atender a todos os pontos de demanda, enquanto no segundo, busca-se maximizar o número de pontos de atendimento cobertos, dado um número restrito de facilidades.

Nos modelos $p$-Centro, as instalações (facilidades) devem ser localizadas em uma rede para minimizar a máxima distância entre os pontos de atendimento e uma instalação $(p=1)$ ou os pontos de atendimento e a respectiva instalação mais próxima $(p>1)$ (DUBKE, 2006). Mladenović et al. (2003) destacam que problemas de localização de unidades do corpo de bombeiros ou de centrais de ambulâncias, em que, a distância da facilidade e seu ponto de atendimento devem ser mínimas, são aplicações típicas de modelos $p$-Centro.

Já os modelos $p$-Medianas buscam minimizar o custo total relacionado (instalação das facilidades e atendimento, ou transporte aos pontos de demanda); nesse caso, o custo de atendimento está, normalmente, associado à distância entre o ponto de atendimento e a facilidade mais próxima (SENNE et al., 2005). Segundo Antiqueira et al. (2012), Chaves et al. (2007) e Diaz e Fernandez (2006) esses modelos podem ser subdivididos em $p$-Medianas Capacitado (capacidade de atendimento limitada) e Não-Capacitado.

A aplicabilidade de PLFs é amplamente mostrada na literatura em diversas áreas: localização de centros de distribuição (MAPA et al., 2006; JIA et al., 2007); serviços de saúde (STERN et al., 1995; CHU e CHU, 2000; BROTNORNE et al., 2003; TAKEDA et al., 2004; JARDIM et al., 2004); serviços públicos (LIMA, 2003; NDIAVE e ALFARES, 2008); educacional (PIZZOLATO e SILVA, 1997; DUTRA, 1998; LOBO, 2003; TEIXEIRA e ANTUNES, 2008); e também na área militar (CHANG et al., 2007; IŞILAK, 2002; MARTIN, 1999; TEAGUE, 1994).

Dessa forma, apresentam-se a seguir, com mais detalhes, algumas aplicações de PLF na área militar. Na abordagem de Chang et al. (2007), o PLF estudado envolveu a quantificação ideal de instalações e suas respectivas 
localizações para Area Processing Centers (APCs), que são centros de processamento de informações do Exército dos EUA. Assim, o objetivo do PLF, nesse caso, foi reduzir o custo total de instalações e a infraestrutura de rede para garantir a segurança das informações. Foi aplicado um modelo de programação linear inteira para esse problema.

Işilak (2002) aplicou a teoria de PLF para combinar de forma ideal, os esforços de distribuição e posicionamento de produtos na rede de distribuição existente para o Exército da Turquia em um processo de investigação do número ideal de depósitos abertos e suas localizações na rede de distribuição. Nesse caso utilizou-se um modelo de programação linear inteira mista.

Martin (1999) desenvolveu um modelo de programação linear inteira mista para auxiliar o Departamento de Defesa dos EUA na decisão de quais locais deveriam se instalar estações de recrutamento; além disso, os recrutados deveriam ser alocados a essas instalações. O modelo foi aplicado de duas maneiras distintas: na primeira, a meta foi obter o menor custo possível; e na segunda, maximizar o número de recrutas incluindo restrições orçamentárias no modelo.

O estudo de Martin (1999) apresentou o modelo com dados das regiões metropolitanas de Denver e Boston nos EUA. Como resultados, quando comparado com as configurações atuais de estações e recrutados, o modelo mostrou que seria possível uma estimativa de redução de custos de 10 a $32 \%$ ou aumento de produtividade (número de recrutados) de 2 a $8 \%$.

Por último, Teague (1994) abordou a localização de estações de recrutamento, com recrutadores da Ativa e Reserva. O objetivo desse modelo foi maximizar uma função de produção descrita pelo número de recrutas para cada Código Postal, em um modelo não-linear de programação inteira, descrita por uma distribuição de Poisson.

\section{MODELO MATEMÁTICO PARA O PL-AOMPP}

O PL-AOMPP consiste em determinar quais OM deverão ser utilizadas (processo de localização) e de forma simultânea fazer a alocação dessas OM aos postos de pesquisa que realizarão as contagens volumétricas classificatórias e pesquisas de origem e destino. Entretanto, cada OM apresenta uma capacidade 
relacionada ao número de pelotões, de forma que, o modelo matemático desenvolvido deve considerar os aspectos operacionais e de efetivos em pelotões das OM.

De maneira prática, devido às limitações de efetivos e a outras necessidades operacionais, cada posto de pesquisa pode ser atendido por uma tropa valor pelotão. Dessa forma, a $\mathrm{OM}$ deve atender aos postos de pesquisa que estão inseridos dentro da área abrangida por seu Comando Militar de Área. A Figura 1 apresenta a distribuição territorial das 221 OM disponíveis e dos 300 postos de pesquisa a serem atendidos.

Figura 1 - Distribuição das OM e postos de pesquisa no território nacional

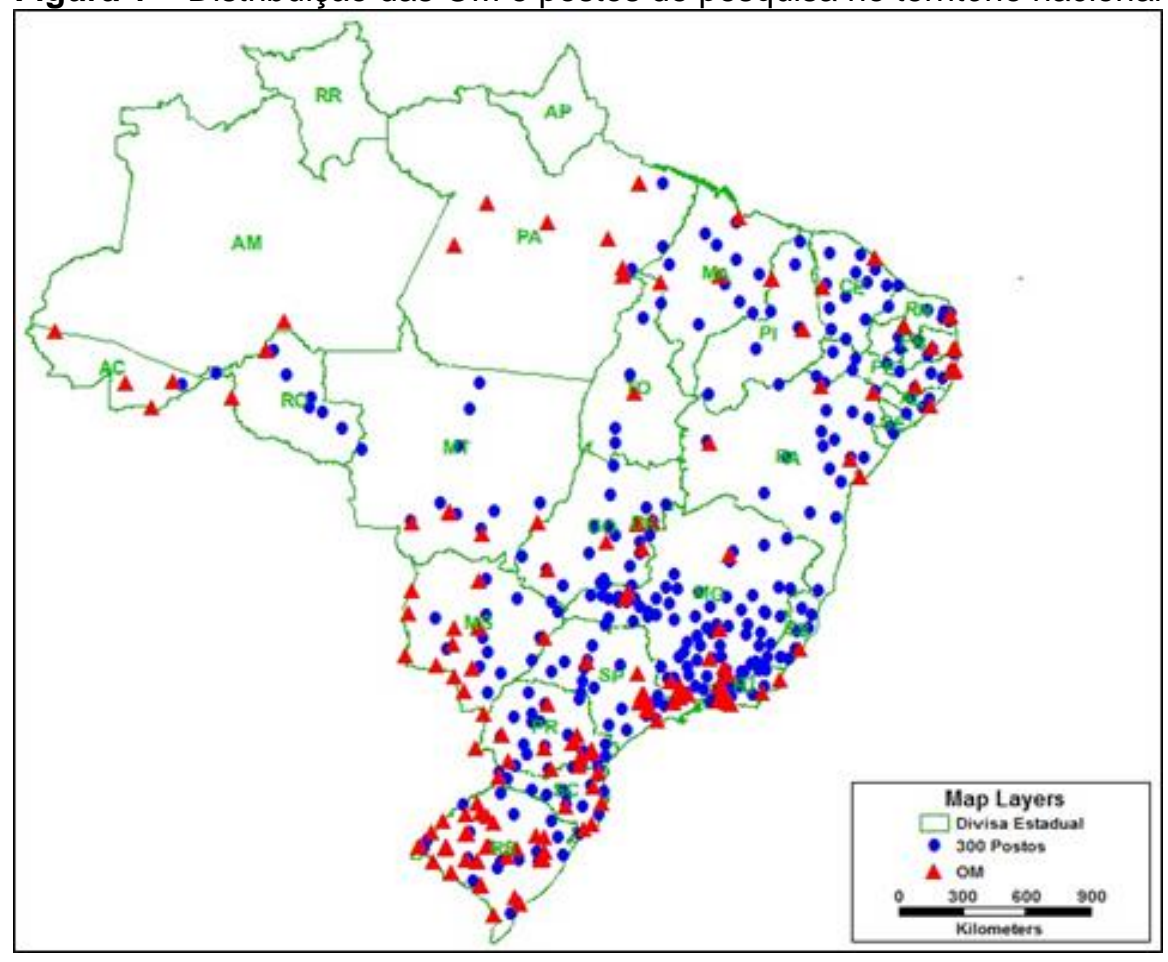

No campo militar, no âmbito do Exército Brasileiro, cada Comando Militar de Área está responsável por uma determinada região do território brasileiro. Deve-se evitar associar uma OM subordinada a um dado Comando Militar de Área a uma região dentro da área de responsabilidade de outro Comando Militar de Área. Por outro lado, cada OM apresenta uma capacidade relacionada ao número de pelotões disponíveis para emprego. 
Assim, seja $O$ o conjunto de todas as OM habilitadas para utilização, $R \quad 0$ conjunto de todas as regiões disponíveis e $P$ o conjunto que engloba todos os postos de pesquisa. Desta maneira, considere que:

- $x_{\sigma p} \in\{0,1\}$ - representa uma variável binária. Se $x_{\sigma p}=1$, a OM $\sigma \in O$ deve ser alocada ao posto $p \in P$, caso contrário, $x_{\sigma p}=0$;

- $y_{\sigma} \in\{0,1\}$ - representa uma variável binária. Se $y_{\sigma}=1$, a OM $\sigma \in O$ deve ser utilizada, caso contrário, $y_{\sigma}=0$;

- $\kappa_{\sigma}$ - é a capacidade associada, em número de pelotões, que a OM $\sigma \in O$ pode ceder para as pesquisas de tráfego;

- $p^{r}$ - considera que cada posto de pesquisa $p \in P$ está associado (instalado) a uma região $r \in R$. Isso torna-se importante devido às características de cada OM que, além da capacidade em quantidade de pelotão, possui restrições de atendimento à determinadas regiões;

- $\sigma^{r}$ - representa a região $r \in R$ da $O M \sigma \in O$;

- $U_{p}$ - é o conjunto de OM candidatas a servir o posto $p \in P$, tal que, $U_{p} \subseteq O$. C conjunto $U_{p}$ pode ser definido como $U_{p}=\left\{\sigma \in O / \sigma^{r}=p^{r}\right\}$;

- $t_{\sigma p}$ - é o tempo de deslocamento entre a $\mathrm{OM} \sigma \in O$ e o posto de pesquisa $p \in P$, tal que, $t_{o p} \geq 0$

- $M A X_{O M}$ - número máximo de OM a serem utilizadas. Por questões de disponibilidade de pessoal, o exército não pode ceder mais que uma dada quantidade de oficiais, que precisa ser convertida em quantidade de OM;

- dummy - denomina-se como dummy uma OM fictícia com capacidade infinita que tem como objetivo adequar o modelo matemático caso a disponibilidade de OM e suas respectivas capacidades não sejam capazes de atender a todos os postos de pesquisa previstos; e 
- $\gamma$ - é um parâmetro que representa um custo (penalidade) atribuída ao uso da OM denominada dummy. $\mathrm{O}$ custo atribuído à $\gamma$ deve ser elevado. Isso se justifica já que, é de interesse que a OM dummy seja utilizada em casos de incapacidade de atendimento pelas OM existentes. Essa incapacidade está relacionada aos parâmetros $M_{A X}$ e $\kappa_{\sigma}, \sigma \in O$, que podem inviabilizar o atendimento de todos os postos de pesquisa. Nos testes computacionais desse modelo, utilizou-se $\gamma=10.000$.

Com isso, para que os pelotões possam atender às demandas dos postos de pesquisa, busca-se então, fazer a alocação (OM - Posto de Pesquisa), tal que, o tempo total de deslocamento da tropa seja minimizado, respeitando as restrições de capacidade. Assim, considerando as informações acima, o modelo matemático, baseado no modelo proposto por Senne et al. (2005) é apresentado a seguir.

Minimizar $z=\sum_{p \in P} \sum_{\sigma \in U_{p}} t_{\sigma p} x_{\sigma p}+\gamma x_{\text {dummy } p}$

Sujeito a:

$$
\begin{array}{ll}
\sum_{\sigma \in U_{p}} x_{\sigma p}+x_{\text {dummy }}=1 & \forall p \in P \\
\sum_{\sigma \in O} y_{\sigma} \leq M A X_{O M} & \\
\sum_{p \in P / \sigma \in U_{p}} x_{\sigma p} \leq y_{\sigma} & \forall p \in P, \sigma \in\left\{\kappa_{\sigma}, \text { dummy }\right\} \\
x_{\sigma p} \in\{0,1\} & \forall \sigma \in O \\
y_{\sigma} \in\{0,1\} & \forall \sigma \in\{O, \text { dummy }\} ; \forall p \in P
\end{array}
$$

A Função Objetivo (1) do problema deve minimizar o tempo total de deslocamento das tropas de cada OM até os respectivos postos de atendimento. $\mathrm{O}$ conjunto de Restrições (2) garante que todos os postos de pesquisa devem ser atendidos, ou alocados à OM dummy. As Restrições (3) garantem que o número máximo de OM será respeitado. As Restrições (4) garantem que, um posto de pesquisa só pode ser atendido por uma OM, se essa OM estiver disponível e for selecionada para atendimento. As Restrições (5) estão relacionadas à capacidade 
de atendimento das OM, portanto, a quantidade de postos atendidos por uma determinada OM deve ser menor que a sua capacidade. Já as Restrições (6) e (7) garantem que as variáveis do problema são binárias.

\section{EXPERIMENTOS COMPUTACIONAIS E ANÁLISE DE RESULTADOS}

Os experimentos computacionais foram realizados em um computador equipado com processador Intel Core i3 com 2,40 GHz e 4 GB de memória RAM. O CPLEX 12.5 (IBM, 2012) foi utilizado para resolver o modelo matemático (1)-(7).

Como mencionado na Seção 1, a nova PNT do DNIT deverá ter 300 postos e contar com o apoio operacional do EB. Após consulta ao setor competente do EB, responsável pela seleção das OM, verificou-se que 221 OM estão disponíveis para realização das pesquisas previstas. Sendo assim, nota-se que $|P|=300$ e $|O|=221$.

Por outro lado, observou-se também que estão disponíveis ao todo 395 pelotões, que o número de pelotões por OM varia entre 1 e 3 , e que a média é de 1,78 pelotões por OM. Conclui-se assim que, teoricamente, seria possível alocar um pelotão a cada posto de pesquisa. Entretanto, o comando militar de cada OM tem impacto direto na relação entre posto de pesquisa e OM.

A Figura 2 apresenta a relação entre OM e Postos de Pesquisa (a) e entre Disponibilidade de Pelotões e Postos de Pesquisa (b), segundo os cinco comandos militares (Norte, Nordeste, Sudeste, Sul e Centro-Oeste). Percebe-se que, ao analisar a Figura 2(a), apenas a região Sul possui OM suficientes para atendimentos de todos os postos regionais, no entanto, a Figura 2(b) mostra que as regiões Nordeste e Sudeste possuem número de pelotões menor que o necessário para atender a todos os postos. A região Sul, nas duas análises, mostra-se como região que, aparentemente, tem menor dificuldade de alocação de OM a postos de pesquisa.

Por outro lado, embora seja possível empregar 221 OM para atuar nas pesquisas, a intenção é utilizar um número menor, dadas outras necessidades operacionais do Exército Brasileiro. Com isso, cenários foram avaliados considerando 30 OM (Cenário 1), 60 (Cenário 2), 90 (Cenário 3), 120 (Cenário 4), 150 (Cenário 5), 180 (Cenário 6) e 221 OM (Cenário 7). 
Figura 2 - Comparação entre: (a) OM e Postos de Pesquisa e (b) Disponibilidade de Pelotões e Postos de Pesquisa

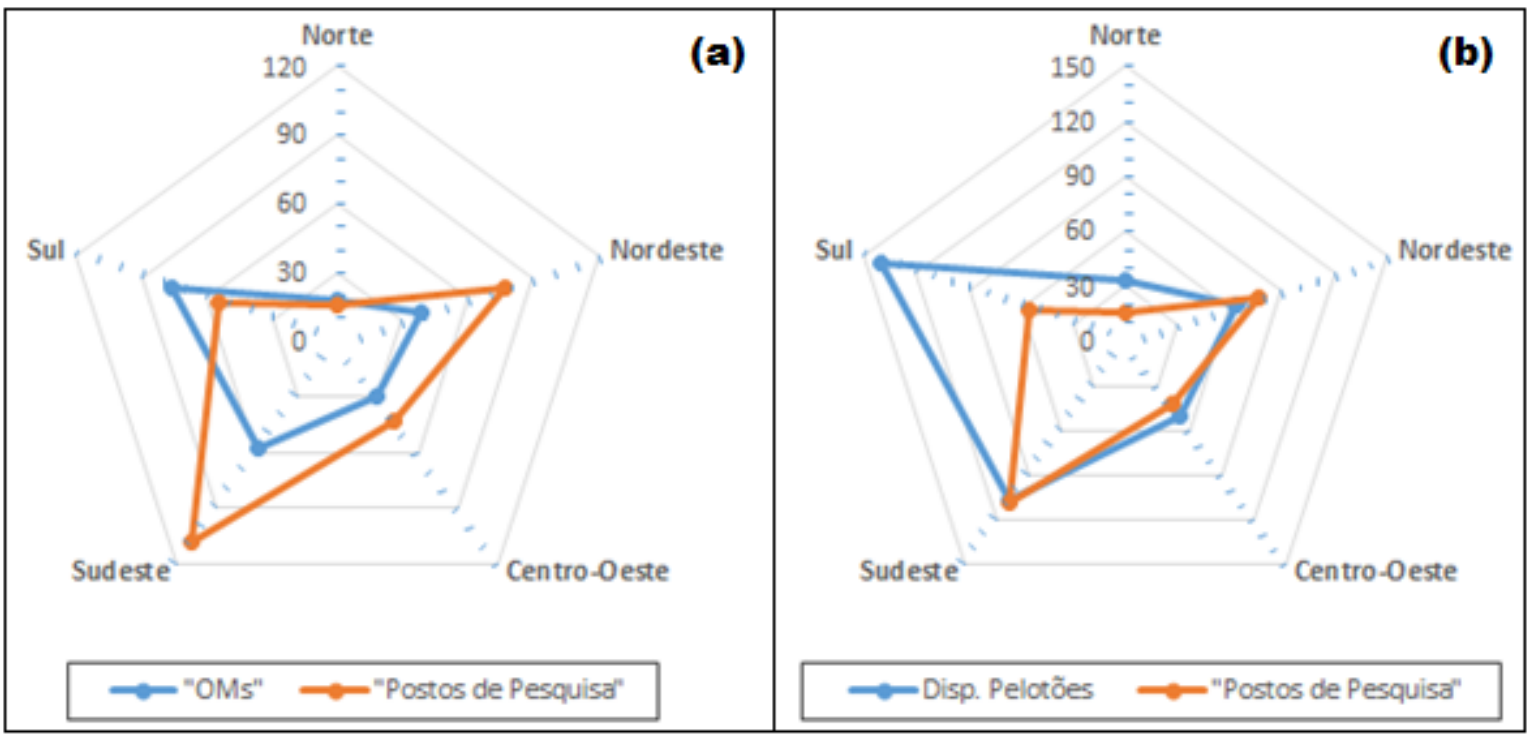

Os resultados estão mostrados na Tabela 1 que está assim dividida: cenário, tempo total de deslocamento dos pelotões, primeiro termo da Função Objetivo (1), total de postos de pesquisa atendidos pelos pelotões, total de OM utilizadas, tempo de processamento do CPLEX, número de soluções obtidas durante o processo de busca, número de iterações do CPLEX e número de nós analisados na árvore de busca (Branch-and-Bound).

Tabela 1 - Resultados dos cenários

\begin{tabular}{|c|c|c|c|c|c|c|c|c|}
\hline \multirow{2}{*}{ 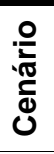 } & \multirow{2}{*}{$\begin{array}{c}\text { Tempo } \\
\text { Deslocamento } \\
\text { (minutos) }\end{array}$} & \multirow[b]{2}{*}{$\begin{array}{c}\text { Postos de } \\
\text { Pesquisa } \\
\text { Atendidos }\end{array}$} & \multirow[b]{2}{*}{$\begin{array}{c}\text { Tempo Médio } \\
\text { Deslocamento } \\
\text { (minutos) }\end{array}$} & \multirow[b]{2}{*}{$\begin{array}{c}\text { Total de OM } \\
\text { Utilizadas }\end{array}$} & \multicolumn{4}{|c|}{ CPLEX } \\
\hline & & & & & $\begin{array}{c}\text { Tempo de } \\
\text { Processamento } \\
\text { (segundos) }\end{array}$ & $\begin{array}{l}\text { Soluções } \\
\text { Inteiras }\end{array}$ & Iterações & $\begin{array}{l}\text { Nós } \\
\text { (B\&B) }\end{array}$ \\
\hline 1 & $6.174,74$ & 90 & 68,61 & 30 & 1,75 & 2 & 1.886 & 0 \\
\hline 2 & $30.729,42$ & 178 & 172,64 & 60 & 5,94 & 5 & 19.128 & 1.640 \\
\hline 3 & $40.731,50$ & 231 & 176,33 & 90 & 12,41 & 14 & 32.546 & 2.389 \\
\hline 4 & $46.102,73$ & 261 & 176,64 & 120 & 12,20 & 4 & 22.475 & 2.451 \\
\hline 5 & $44.822,99$ & 286 & 156,72 & 150 & 2,64 & 6 & 4.583 & 0 \\
\hline 6 & $42.848,17$ & 286 & 149,82 & 172 & 1,09 & 4 & 1.801 & 0 \\
\hline 7 & 42.848 .17 & 286 & 149,82 & 172 & 0,69 & 4 & 1.117 & 0 \\
\hline
\end{tabular}

Pela Tabela 1 verifica-se que à medida que o número de $\mathrm{OM}$ disponíveis aumenta, o tempo total de deslocamento dos pelotões aumenta, bem como o total de postos de pesquisa atendidos. No entanto, ao analisar o tempo médio de deslocamento, é possível notar que para o Cenário 1, com número, consideravelmente, menor de postos atendidos, o tempo médio de deslocamento é 
bastante reduzido, porém, os resultados dos demais cenários indicam que esse parâmetro tende a aumentar um pouco voltando a reduzir na medida que mais postos são atendidos pelas OM.

Por outro lado, percebe-se que o total de OM utilizadas aumenta estabilizando em 172. Verifica-se que embora seja possível utilizar 180 ou 221 OM (Cenários 6 e 7), o resultado encontrado indica que apenas 172 OM devem ser utilizadas para atender 286 postos de pesquisa, ou seja, 14 ficarão sem atendimento. Este resultado mostra que as demais restrições impostas no modelo, principalmente aquelas que se referem aos comandos militares de área, passam a atuar, limitando a utilização de mais OM.

Outro ponto interessante acontece nos Cenários 5 e 6 que possuem 150 e 180 OM disponíveis para atendimento, respectivamente. Embora 286 postos de pesquisa sejam atendidos nos dois cenários, no Cenário 5 as 150 OM disponíveis são utilizadas enquanto o Cenário 6 emprega 172 OM apenas (8 abaixo do total). Assim, o Cenário 6 utiliza $22 \mathrm{OM}$ a mais que o Cenário 5 para reduzir 1.974,82 minutos, ou seja, aproximadamente um dia e meio de deslocamento. Este resultado, apesar de parecer reduzido, é interessante sob o ponto de vista militar uma vez que os veículos utilizados nestas situações apresentam baixa velocidade o que indica longas jornadas e um maior cansaço das tropas envolvidas.

De maneira complementar, apresenta-se também, por meio da Figura 3, a representação gráfica da solução do Cenário 4. A escolha desse cenário para tal demonstração é decorrente da maior proximidade (representatividade) dos parâmetros utilizados com as condições de operação planejadas, à priori, para a realização da nova pesquisa.

Além disso, o Cenário 4 representa um resultado intermediário, relacionado com a quantidade de Postos de Pesquisa atendidos. Com isso, é possível observar que, para esse cenário, a maior quantidade de postos sem atendimento está nos estados que compõem as Regiões Sudeste e Nordeste. Nesses estados, a grande concentração de postos, associado à baixa disponibilidade de OM (comparado com número de postos), já apresentado na Figura 2, pode estar influenciando no desatendimento, tendo em vista a limitação na quantidade de OM utilizadas.

Sob o ponto de vista computacional, nota-se que o CPLEX apresentou um tempo computacional reduzido e que, em alguns casos, não foram necessários abrir 
outros nós, além do nó raiz, durante o processo de busca com o branch-and-bound (Cenários 1, 5, 6 e 7).

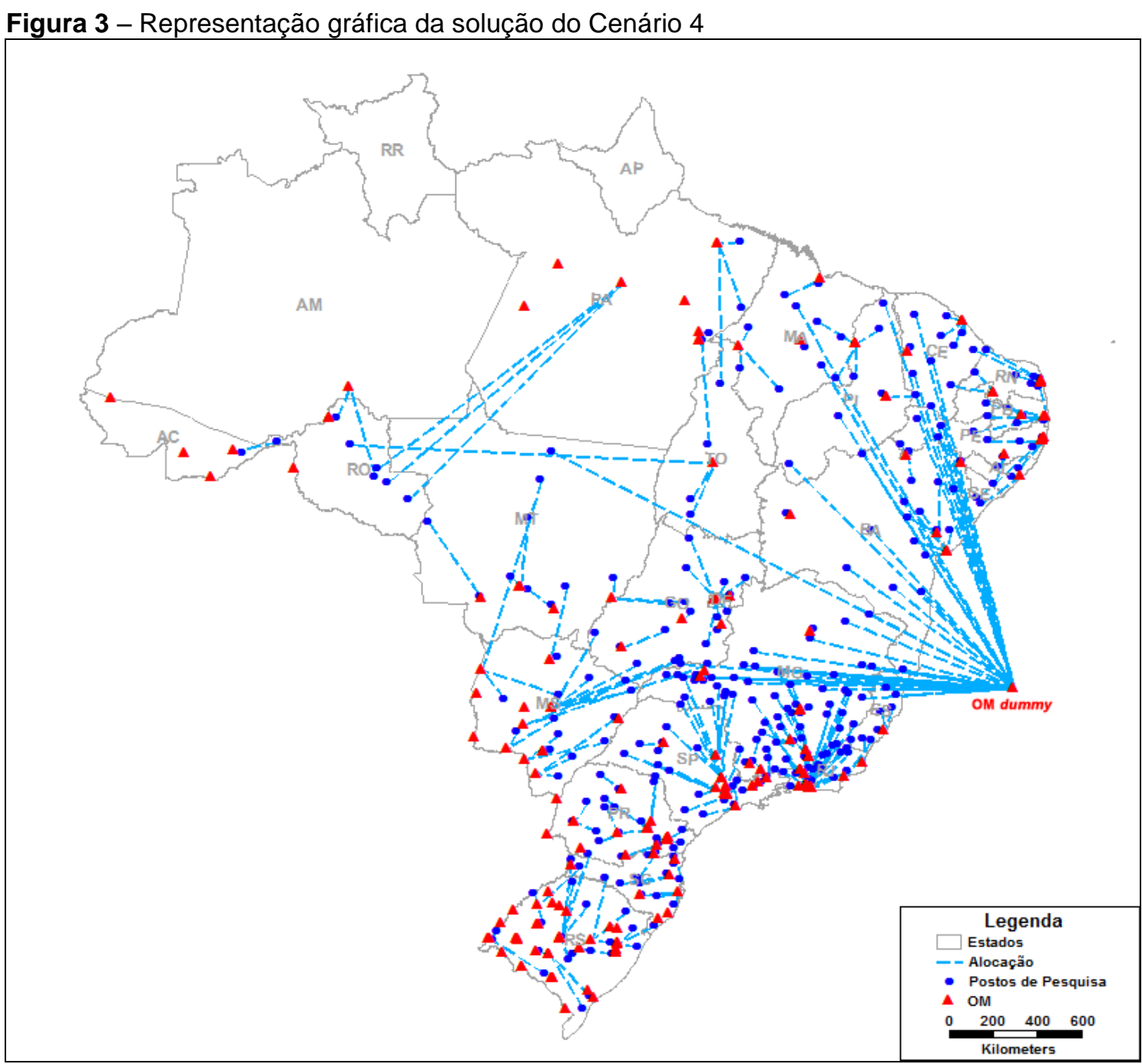

Outra análise importante está relacionada à localização regional dos postos de pesquisa que não foram atendidos por nenhuma OM. Assim, a Figura 4 apresenta, graficamente, a quantidade de postos não atendidos por região e por cenário, em valores unitários e percentuais. Com isso, observa-se que, como era esperado, a região Sul, com exceção do Cenário 1, apresenta os menores percentuais de não atendimento de postos de pesquisa, 26 postos no Cenário 1, 1 no Cenário 2, e nenhum posto sem atendimento nos demais. 
Figura 4 - Quantidade de postos de pesquisa não atendidos por região e por Cenário (unidades e valor percentual)

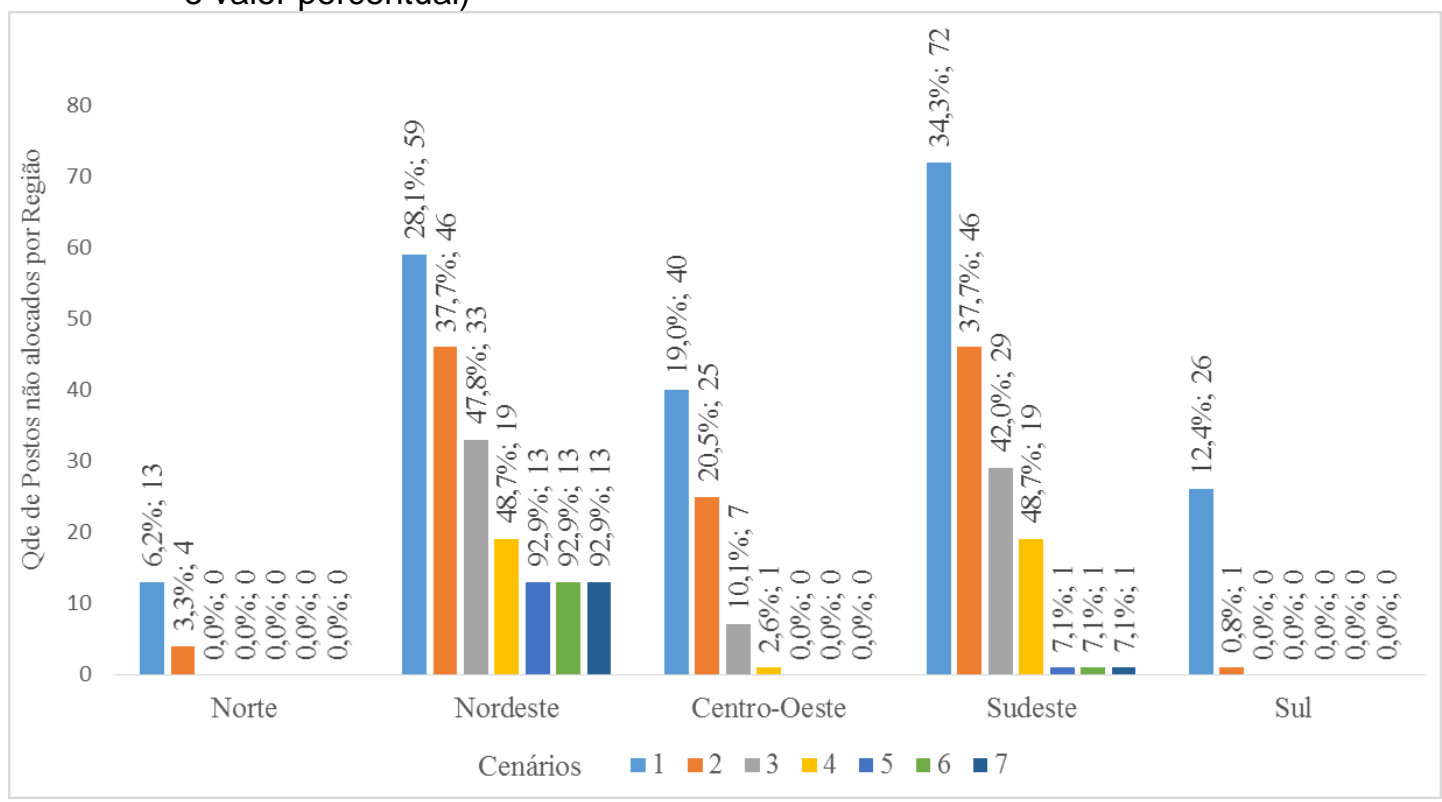

Já a região Norte, assim como na região Sul, nos Cenários 3, 4, 5, 6 e 7, nenhum posto de pesquisa ficou sem atendimento, por outro lado, no Cenário 1 têm 13 postos e no Cenário 2, 4 postos. A região Centro-Oeste apresenta maiores problemas de alocação nos Cenários com 30 e 60 OM disponíveis.

Isso pode se justificar por meio da Figura 1, já que, apesar da grande extensão territorial da região Norte, poucos postos de pesquisa devem ser alocados, além disso, na maioria dos casos, estes apresentam OM próximas para serem utilizadas. Enquanto que, a região Centro-Oeste, possui maior número de postos de pesquisa e, alguns destes, com localizações isoladas (mais distantes da OM mais próxima).

As regiões Nordeste e Sudeste apresentam maiores problemas, quando se analisa os postos não alocados por região. Nos cenários mais restritivos (30, 60, 90 e 120 OM disponíveis), juntas, essas regiões somam, na pior das hipóteses (Cenário 1), $62,4 \%$, chegando a obter $97,4 \%$ no Cenário 4. Já nos Cenários com maior disponibilidade de OM (150, 180 e 221 OM), a região Nordeste apresenta os piores resultados, ou seja, neles $92,9 \%$ dos postos não alocados estão presentes nessa região.

Novamente, a Figura 2 pode justificar tal comportamento, visto que, apesar na relação entre $\mathrm{OM}$ disponíveis e postos a serem atendidos, ser a pior, para a região Sudeste, quando a comparação é relativa à quantidade de pelotões disponíveis por 
posto, a região Nordeste novamente apresenta os piores resultados, com capacidade de pelotões insuficientes para atendimento de todos os postos de pesquisa.

\section{CONSIDERAÇÕES FINAIS}

Este estudo apresentou uma proposição de um modelo matemático para localização e alocação de OM a postos de pesquisa de tráfego no território brasileiro. A proposta do modelo fez uso de pesquisas de tráfego desenvolvidas pelo DNIT em parceria com o Exército Brasileiro (2005, 2008 e 2011), mas principalmente, na pesquisa prevista para ser realizada nos anos de 2015 e 2016.

Com isso, de acordo com informações preliminares obtidas, relacionadas aos parâmetros operacionais das pesquisas anteriores, quantidades e localização de OM, suas respectivas capacidades em número de pelotões e o número de postos previstos para a nova pesquisa, sete cenários de aplicação do modelo foram criados. Em cada um deles, basicamente, foram alteradas as disponibilidades de OM para as pesquisas $(30,60,90,120,150,180$ e 221 OM disponíveis para realização das pesquisas).

Como resultado foi possível realizar uma análise quantitativa para cada cenário, principalmente, relacionada ao número efetivo de $\mathrm{OM}$ utilizadas e à quantidade de postos atendidos. Por consequência, tornou-se viável uma análise dos postos não atendidos, em quantidade e também, por localização regional, indicando as regiões que podem apresentar deficiências de atendimento dos postos de pesquisa.

Nas condições apresentadas, que se pretende atender a todos os postos de pesquisa com no máximo $120 \mathrm{OM}$, verificou-se que alguns postos ficarão sem atendimento, dessa forma, sugere-se: (1) reavaliar capacidades das OM; (2) possibilidade de utilização de OM em postos localizados em comandos de área diferentes; ou (3) dividir a pesquisa de CVC e O/D em fases, o que pode ajudar a garantir atendimento dos postos e reduzir custos com reutilização de uma mesma OM em períodos distintos.

Como limitação deste trabalho, aponta-se o uso de dados estimados, já que a pesquisa prevista para 2015/2016 ainda não está totalmente definida. 


\section{AGRADECIMENTOS}

Os autores agradecem à Coordenação de Aperfeiçoamento de Pessoal de Nível Superior (CAPES) e ao Conselho Nacional de Desenvolvimento Científico e Tecnológico (CNPq) pelo suporte financeiro para desenvolvimento deste trabalho.

\section{REFERÊNCIAS}

ANTIQUEIRA, L. S. de; PEREIRA, E. C.; MACHADO, C. M. dos S. Problema de localização de facilidades aplicado ao serviço de estacionamento rotativo. In: Encontro Nacional de Engenharia de Produção (ENEGEP), 32, 2012, Bento Gonçalves. Anais... Bento Gonçalves: ABEPRO, 2012.

ARAKAKI, R. G. I. Heurística de localização-alocação para problemas de localização de facilidades. $79 \mathrm{f}$. Tese (Doutorado) - Computação Aplicada, Instituto Nacional de Pesquisas Espaciais - INPE, São José dos Campos, 2003.

BALLOU, R. H. Gerenciamento da cadeia de suprimentos: planejamento, organização e logística empresarial. São Paulo: Bookman, 2001.

BRANDEAU, M. L.; CHIU, S. S. Overview of representative problems in location research. Management Science, n. 35, v. 6, p.645-674, 1989.

http://dx.doi.org/10.1287/mnsc.35.6.645

BROTNORNE, L.; LAPORTE, G.; SEMET F. Ambulance location and relocation models. European Journal of Operational Research, v. 147, p. 451-463, 2003. http://dx.doi.org/10.1016/S0377-2217(02)00364-8

CHAVES, A. A.; CORREA, F. A.; LORENA, L. A. N. Clustering search heuristic for the capacitated p-median problem. In: Innovations in Hybrid Intelligent Systems. Springer Berlin Heidelberg, n. 44, p. 136-143, 2007.

http://dx.doi.org/10.1007/978-3-540-74972-1 19

CHU, S. C. K.; CHU, L. A modeling framework for hospital location and service allocation. International Transactions in Operational Research, v. 7, p. 539-568, 2000. http://dx.doi.org/10.1111/j.1475-3995.2000.tb00216.x

CHUANG, P. T. Combining the analytic hierarchy process and quality function deployment for location decision from a requirement perspective. The International Journal of Advanced Manufacturing Technology, n. 18, p. 842-849, 2001.

http://dx.doi.org/10.1007/s001700170010

CHANG, S. J. F.; PATEL, S. H.; WITHERS J. M. An optimization model to determine data center locations for the army enterprise. MILCOM 2007 - IEEE Military Communications Conference, Orlando, FL, USA, p. 1-8, 2007. http://dx.doi.org/10.1109/MILCOM.2007.4455124

CPLEX, T. M. versão 12.5 (2012). IBM ILOG, Sunnyvale, California, 2011. 
CRAINIC, T.; LAPORTE, G. Planning Models for Freight Transportation. European Journal of Operational Research, n. 97, p. 409-438, 1997.

http://dx.doi.org/10.1016/S0377-2217(96)00298-6

DASKIN, M. S. Network and discrete location: models, algorithms, and applications. New York: Wiley Interscience, 1995. http://dx.doi.org/10.1002/9781118032343

DIAZ, J. A.; FERNANDEZ, E. Hybrid scatter search and path relinking for the capacitated pmedian problem. European Journal of Operational Research, n. 169, p. 570-585, 2006. http://dx.doi.org/10.1016/i.ejor.2004.08.016

DEPARTAMENTO NACIONAL DE INFRAESTRUTURA DE TRANSPORTES (DNIT). Plano Nacional de Contagem de Trânsito. Disponível em:

<http://www1.dnit.gov.br/rodovias/contagem/plano_contagem.htm> Acesso em: 17 abr. 2015.

DUBKE, A. F. Modelo de localização de terminais especializados: um estudo de caso em corredores de exportação da soja. Tese (Doutorado em Engenharia de Produção) Pontifícia Universidade Católica - PUC. Rio de Janeiro/RJ. 177p, 2006.

DUTRA, N. G. S. Planejamento de uma rede escolar municipal para reduzir custos de deslocamento. Dissertação (Mestrado em Engenharia) - Escola de Engenharia de São Carlos, Universidade de São Paulo, São Carlos, 1998.

GALVÃO, F. A.; GUALDA, N. D. F.; CUNHA, C. B. An Application of the Analytic Hierarchy Process (AHP) for Locating a Distribution Center. In: International Symposium on the Analytic Hierarchy Process (ISAHP), 2003, Bali. Anais... Bali: 2003. Disponível em: <http://www.isahp.org/2003Proceedings/paper/p33.pdf>. Acesso em: 15 jul. 2015.

DREZNER, Z.; HAMACHER, H. W; Facility location: applications and theory. Berlin: Springer Science \& Business Media, 458 p., 2002.

HURTER, A. P.; MARTINICH, J. S. Facility location and the theory of production. Boston: Kluwer, 1989. http://dx.doi.org/10.1007/978-94-009-2518-2

IŞILAK, A. S. Modeling location-allocation of military items to the depots without branch classification. Tese (Doutorado) - Bilkent University, 2002.

JARDIM, R. X.; CONCEIÇÃO, S. V.; CARVALHO, C. R. V. Localização estratégica para o serviço de atendimento móvel de urgência na região metropolitana de Belo Horizonte. In: Encontro Nacional de Engenharia de Produção (ENEGEP), 24, 2004, Florianópolis. Anais... Florianópolis: ABEPRO.

JIA, H.; ORDÓÑEZ, F.; DESSOUKV, M. M. Solution approaches for facility location of medical supplies for large-scale emergencies. Computers \& Industrial Engineering, v. 52, p. 257-276, 2007. http://dx.doi.org/10.1016/i.cie.2006.12.007

KLOSE, A.; DREXL, A. Facility location models for distribution system design. European Journal of Operational Research, v. 162, n. 1, p. 4-29, 2005.

http://dx.doi.org/10.1016/j.ejor.2003.10.031

KRARUP, J.; PRUZAN, P. M. Ingredients of Location Analysis. In: Mirchandani, P.B. e Francis, R.L. (Eds) Discrete Location Theory, John Wiley, New York, pp.1-54, 1990. 
LIMA, R. S. Bases para uma metodologia de apoio à decisão para serviços de educação e saúde sob a ótica dos transportes. Tese (Doutorado) - Escola de Engenharia de São Carlos, Universidade de São Paulo, São Carlos, 2003.

LOBO. D. S. Dimensionamento e otimização locacional de unidades de educação infantil. Tese (Doutorado) - Programa de Pós-graduação em Engenharia de Produção, Universidade Federal de Santa Catarina, Florianópolis, 2003.

LOPEZ, R. A.; HENDERSON, N. R. The determinants of location choices for food processing plants. Agrobusiness, v. 5, p. 619-632, 1989.

http://dx.doi.org/10.1002/1520-6297(198911)5:6<619::AID-AGR2720050607>3.0.CO;2-A

MAPA, S. M. S. Localização-Alocação de Instalações com Sistema de Informações Geográficas e Modelagem Matemática. Dissertação (Mestrado em Engenharia de Produção) - Universidade Federal de Itajubá - UNIFEI, Itajubá/MG, 2007.

MAPA, S. M. S.; LIMA, R. S.; MENDES, J. F. G. M. Localização de instalações com o auxílio de Sistema de informações Geográficas (SIG) e modelagem matemática. In: Encontro Nacional de Engenharia de Produção (ENEGEP), 26, 2006, Fortaleza. Anais... Fortaleza: ABEPRO.

MARTIN, P. E. A. Multi-service location-allocation model for military recruiting. Dissertação (Mestrado em Pesquisa Operacional) - Naval Postgraduate School, Monterey/CA, 1999.

MINISTÉRIO DOS TRANSPORTES (MT); DEPARTAMENTO NACIONAL DE INFRAESTRUTURA DE TRANSPORTES (DNIT). Pesquisa nacional de tráfego. planejamento para realização das coletas de tráfego de campo e treinamento dos militares que atuarão como técnicos responsáveis pelos postos de pesquisa rodoviária - Tomo 1. Brasília, 2011.

MLADENOVIĆ, N.; LABBÉ, M.; HANSEN, P. Solving the p-Center Problem with Tabu Search and Variable Neighborhood Search. Networks, v. 42, n. 1, p. 48-64, 2003. http://dx.doi.org/10.1002/net.10081

NDIAVE, M.; ALFARES, H. Modeling health care facility location for moving population groups. Computers \& Operations Research v. 35, p. 2154-2161, 2008. http://dx.doi.org/10.1016/j.cor.2006.09.025

NOGUEIRA, T. A. F. M. P. Localização de Unidades de Fornecimento de Gás Natural na Rede Primária de Gasodutos. Tese (Doutorado em Engenharia Electrotécnica e de Computadores) - Universidade de Trás-os-Montes e Alto Douro, Vila Real, Portugal. 281 p, 2007.

PIZZOLATO, N. D.; SILVA, H. B. F. The Location of Public Schools: Evaluation of Practical Experiences. International Transactions in Operational Research, v. 4, n. 1, p. 13-22, 1997. http://dx.doi.org/10.1111/i.1475-3995.1997.tb00058.x

ROMERO, B. C. Análise de Localização de Plataformas Logísticas: Aplicação ao Caso do ETSP - Entreposto Terminal São Paulo da CEAGESP. Dissertação (Mestrado em Engenharia de Sistemas Logísticos) - Escola Politécnica da Universidade de São Paulo POLI/USP, São Paulo/SP, 143 p, 2006. 
SENNE. E. L. F.; LORENA, L.A.N.; PEREIRA, M. A. A Branch-and-price approach to pmedian location problems. Computers \& Operations Research, v. 32, n. 6, p. 1655-1664, 2005. http://dx.doi.org/10.1016/i.cor.2003.11.024

STERN, Z. S.; MEHREZ, A.; TAL, A.; SHEMUEL, B. The location of a hospital in rural region: the case of the Nevev. Location Science, v. 3, n. 4, p. 255-266, 1995. http://dx.doi.org/10.1016/0966-8349(96)00002-2

TAKEDA R. A.; WIDMER, J. A.; MORABITO, R. Aplicação do Modelo Hipercubo de Filas para Avaliar a Descentralização de Ambulâncias em um Sistema Urbano de Atendimento Médico de Urgência. Pesquisa Operacional, v. 24, n. 1, p. 39-71, 2004.

http://dx.doi.org/10.1590/S0101-74382004000100004

TEAGUE, M. J. An Optimal Allocation of Army Recruiting Stations With Active and

Reserve Recruiters. Dissertação (Mestrado em Pesquisa Operacional) - Naval

Postgraduate School, Monterey/CA, 1994.

TEIXEIRA, J. C.; ANTUNES, A. P. A. Hierarchical location model for public facility planning. European Journal of Operational Research, v. 185, p. 92-104, 2008.

http://dx.doi.org/10.1016/j.ejor.2006.12.027

YANG, J.; LEE, H. A AHP Decision Model for Facility Location Selection. Facilities, n. 15, p. 241-254, 1997. http://dx.doi.org/10.1108/02632779710178785

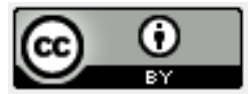

Artigo recebido em 14/08/2015 e aceito para publicação em 17/05/2016

DOI: http://dx.doi.org/ 10.14488/1676-1901.v16i2.2125 